\title{
Disección aórtica tipo A: Resultados operatorios y seguimiento a mediano plazo
}

\author{
RAFAEL SELMAN A. ${ }^{1,2}$, ANDRÉS KURSBAUM E. ${ }^{2}$, MATÍAS UBILLA S. ${ }^{1}$, \\ EDUARDO TURNER G. ${ }^{1,2}$, CHRISTIAN ESPINOZA S. ${ }^{2}$, JUAN ESPINOZA H. ${ }^{2}$, \\ RODRIGO GONZÁLEZ F. ${ }^{2}$, MAURICIO VILLAVICENCIO T. ${ }^{2}$, \\ ELIZABETH VALLADARES J. ${ }^{\text {a }}$, LORENZO NARANJO T. ${ }^{2}$, ERHARD OPPLIGER P. ${ }^{2}$
}

'Servicio de Cirugía Cardíaca Hospital DIPRECA.

${ }^{2}$ Servicio de Cirugía Cardíaca Instituto Nacional del Tórax (INT).

anterna de Medicina, Universidad de Santiago.

Recibido el 8 de marzo de 2010, aceptado el 9 de agosto de 2010

Correspondencia a:

Dr. Rafael Selman A JM Infante 717, Providencia.

Fono (56 2) 5754986 Fax (56 2)

E-mail: rselman@manquehue. net

\section{Surgical results among 100 patients with type A aortic dissection. Retrospective review}

Background: Tipe A aortic dissection involves the ascending aorta and has high mortality rates without surgical treatment. Aim: To report the results of surgical treatment of type A aortic dissection. Material and Methods: Retrospective review of medical records of 100 patients aged 17 to 78 years ( $73 \%$ males) operated between January 2000 and August 2008, for type A aortic dissection. Follow up was performed with telephone interviews and review of national death records. Results: Eighty three percent of patients had an acute dissection. Operative mortality was 27 and 20\% for patients with acute and chronic dissection, respectively. Mortality was 50\% among patients aged 70 years or more, compared with $21 \%$ among their younger counterparts, The most common complication was bleeding that required a new surgical procedure in $18 \%$ of patients. Actuarial survival was $70 \%$ at five years. Cardiovascular problems caused the death of two of the nine patients that died during follow up. Conclusions: Surgical mortality among patients with type A aortic dissection was higher among patients with acute episodes and those aged 70 years or more.

(Rev Med Chile 2010; 138: 982-987).

Key words: Aortic diseases; Cardiovascular surgical procedures; Fatal outcome.
L a disección aórtica tipo A consiste en el desgarro de la íntima de la aorta torácica ascendente, habitualmente inmediatamente distal a la unión senotubular, con la formación de un canal paralelo al lumen natural denominado lumen falso. Este último se propaga hacia distal en extensión variable y puede producir compresión del lumen verdadero, causando una pseudocoartación de la aorta toráxica o estenosis u obstrucción de ramas que nacen de la aorta al avanzar hacia estas, generando el debilitamiento de la pared y facilitando la ruptura aguda o la formación de un aneurisma en la etapa crónica. El falso lumen también se propaga hacia proximal, causando desprendimiento de las comisuras de la válvula aórtica, lo que genera grados variables de insuficiencia aórtica aguda.

Dado que la disección se propaga a medida que pasan las horas, con riesgo de isquemia visceral o incluso de extremidades inferiores por obstrucción al flujo del lumen verdadero, es muy importante una sospecha diagnóstica para un tratamiento oportuno en todo paciente que debuta con dolor toráxico brusco y transfixiante al dorso, especialmente si dicho dolor es migratorio hacia la región lumbar por propagación de la disección.

El síndrome coronario agudo es su principal diagnóstico diferencial, también se presenta con dolor toráxico, pero éste es más incidioso (no brusco sino de intensidad creciente), habitualmente no 
es transfixiante al dorso ni tampoco migratorio, tiene habitualmente cambios electrocardiográfico tipo isquémicos y puede tener alza enzimática.

Ante una sospecha diagnóstica, debiera realizarse primero una Radiografía de Tórax que entre el 60 al 90\% de los casos es anormal mostrando ensanchamiento del mediastino anterior y superior, confirmando el diagnóstico con una tomografía axial computada (TAC) de tórax y abdomen con contraste o en su defecto un ecocardiograma transesofágico (TEE).

En USA la disección aórtica aguda tiene una incidencia anual de 6,6 casos por millón de habitantes $^{1}$, de ellas, aproximadamente $66 \%$ son de tipo A (comprometen la aorta ascendente), lo que extrapolándolo a la población chilena, habrían unos 70 pacientes anualmente. La incidencia peak es entre la 5 ta y 7 ma década y tres veces más frecuente en hombres.

La disección aórtica tipo A es una patología con alta letalidad sin cirugía, llegando aproximadamente a $50 \%$ a las 48 horas, $75 \%$ a la semana y $95 \%$ al mes ${ }^{1}$. Con cirugía, la mortalidad de disección aguda según el Registro Internacional de Disección Aórtica (IRAD), llega a 26\% y $37 \%$ en pacientes menores y mayores de 70 años ${ }^{2}$.

El objetivo del presente trabajo es evaluar nuestros resultados en término de morbi-mortalidad operatoria y seguimiento a mediano plazo.

\section{Material y Método}

El presente estudio es un análisis retrospectivo de todos los pacientes operados en forma consecutiva por disección aórtica tipo A entre el 1 de enero de 2000 y el 31 de agosto de 2008, en los Hospitales DIPRECA e INT.

El diagnóstico estuvo basado en un cuadro clínico compatible y la obtención de imágenes mediante TAC y/o TEE. Previo al procedimiento, todos los pacientes fueron evaluados mediante TAC helicoidal multicorte con contraste en fase arterial. En los casos dudosos, el estudio preoperatorio se complementó con un TEE. La información se obtuvo del análisis de Fichas Clínicas. Se evaluó sobrevida actuarial según método de KaplanMeier usando base de datos del Registro Civil y entrevistas telefónicas. Se utilizó análisis univariado de factores asociados a morbi-mortalidad según test de Mann Whitney, considerando diferencias estadísticamente significativas si $\mathrm{p} \leq 0,05$.

\section{Técnica Anestésica}

Mezcla de halogenados y narcóticos endovenosos de manera habitual, heparinización sistémica Standard para tiempo de coagulación activado (TCA) > 480 seg, ultrafiltración venosa de rutina para remover mediadores inflamatorios, uso de antifibrinolíticos en forma habitual (Aprotinina 4 a 6 millones UI hasta noviembre 2007 o ácido tranexámico $100 \mathrm{mg} / \mathrm{kg}$ desde noviembre 2007).

\section{Técnica Quirúrgica}

Esternotomía media, canulación arterial femoral/axilar según el caso, canulación venosa única de doble drenaje, paro circulatorio en hipotermia moderada $\left(18-24^{\circ} \mathrm{C}\right)$ para la anastomosis distal, protección cerebral según preferencia del cirujano (sólo hipotermia, retrógrada o anterógrada selectiva). Anastomosis proximal preparando cabo con cola biológica o "Bio Glue" usando refuerzo con Teflón según preferencia de cirujano. Anastomosis distal con aorta "desclampeada" en paro circulatorio, resecando arco aórtico si rasgo de disección se inicia en el arco o éste se encuentra dilatado $(\geq 5 \mathrm{~cm})$.

Uso de tubo de Dacrón microporoso precoagulado para reemplazo de aorta. Tubo valvulado si la válvula aórtica está insuficiente y no es reparable.

\section{Definiciones}

- Disección aórtica aguda: aquella que tiene una data $\leq 2$ semanas desde el inicio de los síntomas al diagnóstico.

- Disección aórtica crónica: aquella que tiene una data $>2$ semanas desde el inicio de los síntomas al diagnóstico.

- Insuficiencia renal preoperatoria: Creatininemia preoperatoria $\geq 2 \mathrm{mg} / \mathrm{dl}$.

- Insuficiencia renal aguda postoperatoria: Duplicación de la creatininemia basal o requerimiento de diálisis.

- Mortalidad operatoria: mortalidad hospitalaria o muertes ocurridas dentro de los primeros 30 días desde la operación.

\section{Resultados}

Entre el 1 de enero del 2000 y el 31 de agosto de 2008 se operaron en forma consecutiva 100 pacientes, $73 \%$ hombres y con edad promedio de 55,43 años (rango 17-78 años), 89 pacientes en el 
INT y 11 en el Hospital DIPRECA. La distribución anual se detalla en la Figura 1 y las comorbilidades se detallan en la Tabla 1; las más frecuentes fueron la hipertensión arterial (77,53\%), insuficiencia valvular aórtica moderada o severa $(69,66 \%)$ y el shock cardiogénico o tamponamiento $(22,47 \%)$. Además, $7,78 \%$ de los pacientes se operaron en insuficiencia renal aguda.

En $83 \%$ de los pacientes la disección aórtica fue aguda. Desde el 2005 se empezó a utilizar canulación axilar derecha de rutina y para el grupo total correspondió a 54\%. Los tiempos quirúrgicos se detallan en la Tabla 2, destacando un tiempo de circulación extracorpórea promedio de 186 minutos y un tiempo de paro circulatorio promedio de 34 minutos.

Las técnicas de protección cerebral se detallan en la Tabla 3, destacando perfusión cerebral anterógrada selectiva en el $51 \%$ de los pacientes, lo que es coincidente con $\%$ similar de pacientes sometidos a canulación axilar.

La morbilidad postoperatoria se detalla en la Tabla 4, destacando que sólo $20,73 \%$ de los pacientes no tuvieron complicaciones y que la complicación más frecuente fue necesidad de reexploración por sangramiento o tamponamiento en 18\% de los pacientes.

La mortalidad operatoria global fue de $26 \%$ (Tabla 5), siendo $50 \%$ y $21,43 \%$ para pacientes $\geq \mathrm{y}<$ de 70 años respectivamente $(\mathrm{p}=0,042)$. La mortalidad operatoria según el procedimiento realizado se detalla en la Tabla 6, destacando una mortalidad operatoria de $12 \%$ en cirugía de reemplazo de aorta ascendente con resuspensión de válvula aórtica, que fue el procedimiento más común.

El seguimiento postoperatorio fue de $100 \%$, con una sobrevida actuarial de $70,1 \%$ a 5 años (Figura 2). Hubo 9 fallecidos de los 74 sobrevivientes,

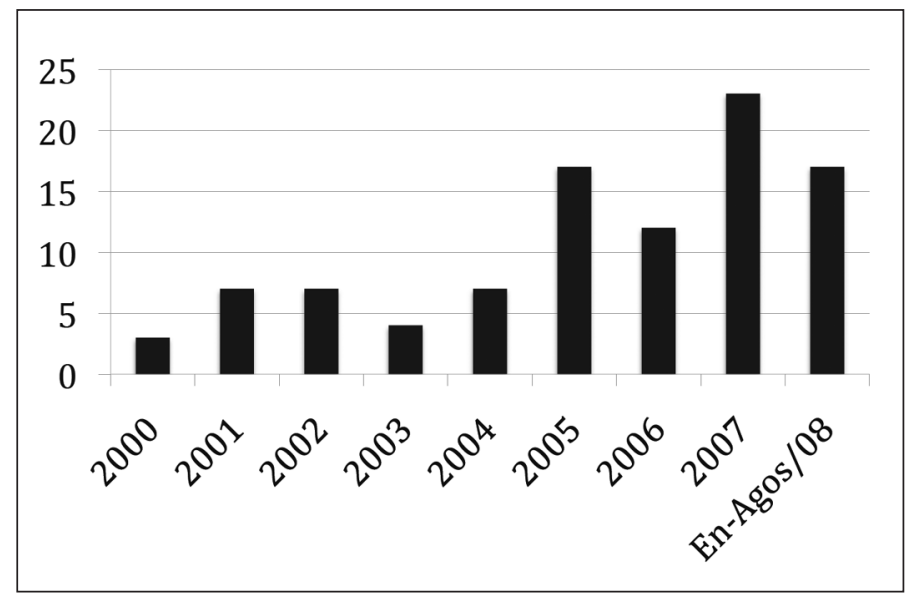

Figura 1. Distribución de casos según períodos.

Tabla 1. Morbilidad preoperatoria

\begin{tabular}{|lrr|}
\hline Morbilidad preoperatoria & n & \% \\
\hline HTA & 69 & 77,53 \\
\hline Diabetes mellitus & 6 & 6,74 \\
\hline Insuficiencia renal* & 7 & 7,87 \\
\hline Cardiopatía coronaria & 4 & 4,49 \\
\hline Válvula aórtica bicúspide & 5 & 5,62 \\
\hline Insuficiencia aórtica mod./severa & 62 & 69,66 \\
\hline Cirugía cardíaca previa & 2 & 2,25 \\
\hline Shock cardiogénico y/o tamponamiento & 20 & 22,47 \\
\hline Insuficiencia arterial aguda & 2 & 2,25 \\
\hline Isquemia mesentérica & 2 & 2,25 \\
\hline Compromiso neurológico & 7 & 7,87 \\
\hline Embarazo tercer trimestre & 2 & 2,25 \\
\hline Síndrome de Marfán & 3 & 3,37 \\
\hline
\end{tabular}

Nota: información de 90 de los 100 pts. ${ }^{*}$ Cretininemia preoperatoria $\geq 2$ $\mathrm{mg} / \mathrm{dl}$.

Tabla 2. Tiempos quirúrgicos

\begin{tabular}{|lcl|}
\hline Variable & $\mathbf{N}^{\circ} \mathbf{F}$ & Promedio (rango) \\
\hline Tiempo CEC (min.) & 76 & $186,34(90-365)$ \\
\hline Tiempo Clamp Ao. (min.) & 79 & $110,52(28-104)$ \\
\hline Paro Circulatorio (min.) & 75 & $34,64(0-88)$ \\
\hline
\end{tabular}

‡ Paciente con información completa. 
Tabla 3. Técnica de protección cerebral

\begin{tabular}{|lcc|}
\hline Tipo protección cerebral & $\mathbf{n}$ & $\mathbf{\%}$ \\
\hline Sólo hipotermia & $7(78)$ & 8,97 \\
\hline Perfusión anterógrada & $40(78)$ & 51,28 \\
\hline Perfusión retrógrada & $28(78)$ & 35,90 \\
\hline Perfusión mixta & $3(78)$ & 3,85 \\
\hline
\end{tabular}

( ): Pacientes en Riesgo.

Tabla 4. Morbilidad postoperatoria

\begin{tabular}{|lcc|}
\hline Variable & $\mathbf{n}$ & $\%$ \\
\hline Sin complicaciones & $16(77)$ & 20,78 \\
\hline Reexploración x sangramiento o tamponamiento & $14(77)$ & 18,18 \\
Arrítmias (AC x FA, Flutter, FV) & $6(77)$ & 7,79 \\
\hline Sepsis & $5(77)$ & 6,49 \\
Accidente cerebrovascular & $4(77)$ & 5,19 \\
Neumonia nosocomial & $4(77)$ & 5,19 \\
Insuficiencia renal aguda* & $4(77)$ & 5,19 \\
Paraplejia postoperatoria & $2(77)$ & 2,59 \\
\hline Misceláneos & & \\
$\quad$ - Reoperación x insuficiencia Ao & $1(77)$ & 1,3 \\
$\quad$ - Síndrome de bajo débito & $1(77)$ & 1,3 \\
$\quad$ - Implante de endoprótesis & $1(77)$ & 1,3 \\
$\quad$ - Hemotórax que requiere drenaje & $1(77)$ & 1,3 \\
\hline
\end{tabular}

( ): Pacientes en Riesgo. *Duplicación de Creatininemia basal o requerimiento de diálisis.

Tabla 5. Mortalidad operatoria

\begin{tabular}{|lrll|}
\hline Mortalidad & $\mathbf{n}$ & $\mathbf{\%}$ & $\mathbf{p}$ \\
Global & $26(100)$ & 26 & \\
$\begin{array}{l}\text { Tipo de disección } \\
\quad \text { - Aguda }\end{array}$ & $23(85)$ & 27,06 & 0,43 \\
$\quad$ - Crónica & $3(15)$ & 20 & \\
Grupo etario & & & \\
$\quad$ - $\geq 70$ años & $8(16)$ & 50 & 0,042 \\
$\quad-\quad 70$ años & $18(84)$ & 21,43 & \\
Período de estudio & & & \\
$\quad$ - 2000-2004 & $8(30)$ & 26,67 & 0,76 \\
$\quad$ - 2005-2008 & $18(70)$ & 25,71 & \\
\hline
\end{tabular}

( ): Pacientes en Riesgo. destacando sólo 2 fallecidos por causas cardiovasculares.

\section{Discusión}

La disección aórtica tipo A es una enfermedad grave, con una alta letalidad sin cirugía. La disección aórtica aguda es una entidad que forma parte del síndrome aórtico agudo, que engloba otras patologías interrelacionadas como el hematoma intramural aórtico y la úlcera aórtica penetrante ${ }^{3}$.

Clásicamente se estima una mortalidad con tratamiento médico exclusivo de aproximadamente $1 \%$ por hora las primeras 48 horas, llegando a $75 \%$ a los 7 días y sobre el $90 \%$ al mes. Por ello, el tratamiento quirúrgico de urgencia es considerado como el Gold Standard, debiéndose actuar tan pronto se hace el diagnóstico.

Estudios más recientes ${ }^{15}$ indican que el riesgo de mortalidad sin cirugía puede no ser tan elevado con tratamiento médico moderno exclusivo o cirugía en diferido, recomendando reconsiderar la cirugía en casos de accidente vascular constituido reciente, comorbilidades serias tales como cáncer, enfermedad pulmonar obstructiva crónica severa, insuficiencia renal aguda o edad muy avanzada ( $\geq$ 80 años) y presentación a la Unidad de Emergencia después de las primeras 48 horas.

La cirugía conlleva una alta mortalidad, llegando a ser de $26 \%$ en el IRAD y $33 \%$ en el Registro Español de Síndrome Aórtico Agudo: RESA ${ }^{4}$. Sin embargo, hay grupos quirúrgicos experimentados que reportan mortalidades entre 10 y $20 \%{ }^{5-11}$, habiendo algunos que reportan mortalidades operatorias inferiores al 10\% ${ }^{12-14}$.

Consideramos que nuestros resultados quirúrgicos en el tratamiento de esta grave patología han sido excelentes, comparables a Centros de Referencia internacionales, teniendo 
Tabla 6. Mortalidad según técnica quirúrgica empleada

\begin{tabular}{|lrrrr|}
\hline Operación & n & \% & $\begin{array}{c}\text { Mortalidad } \\
\text { (\%) }\end{array}$ \\
\hline A.A. sólo & 31 & 31 & $9(29,03)$ \\
\hline A.A. + Resusp. Valv. Ao. & 33 & 33 & $4(12,12)$ \\
\hline A.A. + Arco Ao. & 11 & 11 & $3(27,27)$ \\
A.A. + Valv. Ao. & 5 & 5 & $4(80)$ \\
\hline Tubo valvulado & 15 & 15 & $2(13,33)$ \\
Operación de David & 2 & 2 & $1(50)$ \\
Freestyle + A.A. & 3 & 3 & $2(66,67)$ \\
Procedimiento asociado & & & & \\
$\quad-$ Bypass coronario & 5 & 5 & 1 & $(20)$ \\
$\quad$ - Plastía mitral & 1 & 1 & 0 & \\
\hline
\end{tabular}

Nota: A.A.: Aorta Ascendente.

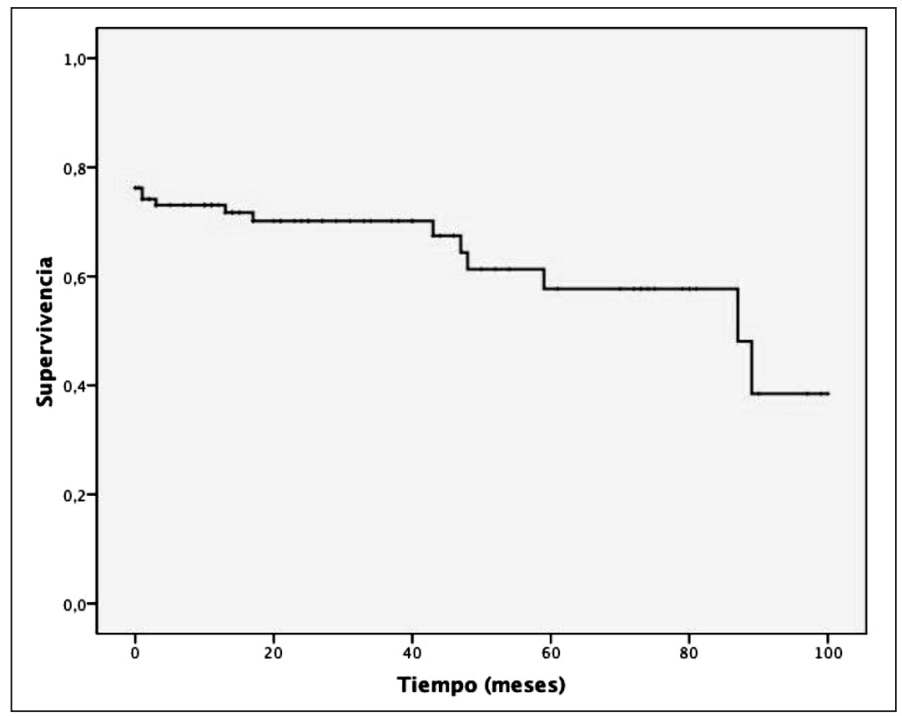

presente la complejidad de nuestros pacientes: $22,47 \%$ de pacientes en shock cardiogénico o tamponamiento preoperatorio, $16 \%$ de pacientes $\geq 70$ años y $7,87 \%$ de pacientes con insuficiencia renal aguda preoperatoria.

La elevada mortalidad observada en pacientes $\geq 70$ años nos indica la necesidad de una selección quirúrgica más estricta para este grupo etario, acorde al estudio recientemente publicado ${ }^{15}$.

La mortalidad alejada $(12,16 \%)$ no estuvo relacionada con la disección en la mayoría de los pacientes (aprox. $90 \%)$, lo que indica que en la mayoría de los pacientes, la cirugía logra ser curativa.

Figura 2. Sobrevida actuarial.

\section{Referencias}

1. Hagan P, Nienaber C, Isselbacher C, Bruckman D, Karavite $\mathrm{D}$, Russman $\mathrm{P}$, et al. The International Registry of Acute Aortic Dissection (IRAD). JAMA 2000; 283: 897-903.

2. Mehta R, O'Gara P, Bossone E, Nienaber C, Myrmel T, Cooper J, et al. Acute Type A Aortic Dissection in the Elderly: Clinical Characteristics, Management and
Outcomes in the Current Era. J Am Coll Cardiol 2002; 40: 685-92.

3. Tsai T, Nienaber C, Eagle K. Acute Aortic Syndromes. Circulation 2005; 312: 3802-13.

4. Evangelista A, Padilla F, López-Ayerbe J, Calvo F, LópezPérez J, Sánchez V, et al. Registro Español del Síndrome Aórtico Agudo (RESA). La mejora en el diagnóstico no se refleja en la reducción de la mortalidad. Rev Esp Cardiol 2009; 62: 255-62. 
5. Halstead J, Meier M, Etz C, Spielvogel D, Bodian C, Wurm M, et al. The Fate of the Distal Aorta After Repair of Acute Type A Aortic Dissection. J Thor Cardiovasc Surg 2007; 133: 127-35.

6. Immer F, Aydin N, Lütolf M, Kragenbühl E, Stalder M, Englberger L, et al. Does Aortic Crossclamping During the Cooling Phase Affect the Early Clinical Outcome Of Acute Type A Aortic Dissection?. J Thor Cardiovasc Surg 2008; 136: 1536-40.

7. Kasui T, Washiyama N, Abul Hasan Muhammad B, Terada H, Yamashita K, Takinami M, et al. Extended Total Arch Replacement for Acute Type A Aortic Dissection: Experience with Seventy Patients. J Thor Cardiovasc Surg 2000; 119: 558-65.

8. Lai D, Miller D, Mitchell R, Oyer P, Moore K, Robbins $\mathrm{R}$, et al. Acute Type A Aortic Dissection Complicated by Aortic Regurgitation: Composite Valve Graft versus Separate Valve Graft versus Conservative Valve Repair. J Thor Cardiovasc Surg 2003; 126: 1978-86.

9. Sabik J, Lytle B, Blackstone E, Mccarthy P, Loop F, Coosgrove D. Long-term Effectiveness of Operations for Ascending Aortic Dissections. J Thor Cardiovasc Surg 2000; 119: 946-62.

10. Narayan P, Rogers C, Davies I, Angelini G, Bryan A.
Type A Aortic Dissection: Has Surgical Outcome Improve with Time?. J Thor Cardiovasc Surg 2008; 136: 1172-7.

11. Estrera A, Garami Z, Miller C, Porat E, Achouh P, Dareshwar J, et al. Acute Type A Aortic Dissection Complicated by Stroke: Can Inmediate Repair be Perform Safely?. J Thor Cardiovasc Surg 2006; 132: 1404-8.

12. Uchida N, Shibamura H, Katayama A, Shimada N, Sutoh M, Ishihara H. Operative Strategy for Acute Type A Aortic Dissection: Ascending Aortic or Hemiarch versus Total Arch Replacement with Frozen Elephant Trunk. Ann Thor Surg 2009; 87: 773-7.

13. Mori Y, Hirose H, Takagi H, Umeda Y, Fukumoto Y, Shimabukuro K, et al. Aortic Arch Repair for Stanford Type A Aortic Dissection with Distal Anastomosis to the Proximal Level of the Distal Aortic Arch. J Thor Cardiovasc Surg 2003; 126: 415-9.

14. Kimura M, Tanaka M, Kawahito K, Yamagushi A, Ino T, Adashi H. Influence of Patent False Lumen on Longterm Outcome After Surgery for Acute Type A Aortic Dissection. J Thor Cardiovasc Surg 2008; 136: 1160-6.

15. Elefteriades J A, Feldman M. Acute Type A Aortic Dissection: Surgical Intervention For All: Con. Cardiology Clinics 2010; 28: 325-32. 